\title{
Modelo de gestión de proyectos de desarrollo tecnológico y vinculación de un centro de I\&DT universitario
}

\author{
Technology Development Project Management \\ and Linking Model for a University R\&D Center \\ Vega-González L.R. \\ Coordinación de Vinculación y Gestión Tecnológica \\ Centro de Ciencias Aplicadas y Desarrollo Tecnológico, CCADET-UNAM \\ Email:lrvg@servidor.unam.mx
}

Información del artículo: recibido: enero de 2009, aceptado: junio de 2009

\begin{abstract}
Resumen
Las actividades que conforman la Gestión Tecnológica de Proyectos (GT de P) son muy diversas y especializadas; sin embargo, aparentemente carecen de valor para los usuarios de estos servicios de gestión en el medio académico. Por esta razón, un primer paso en la búsqueda de elementos que contribuyan a mejorar la percepción y a definir el valor intangible que aporta la vinculación y la gestión tecnológica de los proyectos patrocinados, es la construcción de un modelo empírico de Gestión Tecnológica de Proyectos (GT de P). En este trabajo se muestra un primer modelo de gestión, logrado a través de la recopilación de información y del análisis de la gestión de los proyectos patrocinados en los que ha participado la Coordinación de Vinculación del Centro de Ciencias Aplicadas y Desarrollo Tecnológico (CCADET) de la Universidad Nacional Autónoma de México (UNAM). La estructura del modelo sugiere que la calidad de los resultados de los proyectos de desarrollo tecnológico (DT), no sólo depende de la eficiencia y eficacia del equipo de desarrollo, sino también de la habilidad para aplicar diversas técnicas de gestión. Ante las evidencias se intuye que tal vez la mejor forma de preparación para realizar este trabajo es "aprender haciendo" (learning by doing), participando en la ejecución en las diferentes fases de gestión de muchos proyectos diferentes. Esto hace las veces de un "laboratorio de experimentación" real que permite atacar y resolver diversas situaciones problemáticas. El Modelo de GT de P presentado fue integrado a partir de la gestión de aproximadamente un centenar de proyectos en un periodo de cuatro (4) años.
\end{abstract}

\section{Descriptores}

- circuitos de negociación

- gestión tecnológica de proyectos

- vinculación 


\begin{abstract}
The activities for the Management of Technology Development Projects (MTDP) and linking are diverse and very specialized; nevertheless apparently they have low value in the academic user's media. For that reason, as a first step to build a better perception and to define the intangible value added to externally financed projects, we build an MTDP and linking empirical model. The model was obtained through the information compilation and the analysis of the technological transfer and projects management office of the Centro de Ciencias Aplicadas y Desarrollo Tecnológico (CCADET) of the National Autonomous University of México (UNAM). The model structure suggests that good quality results for the technology development projects depends on the efficiency of the academic development team, but also on the ability to apply different management techniques. Probably the best way to proceed to develop these abilities is "learning by doing", participating in the execution of many different projects. Using the model is similar to an experimental laboratory for the project management permitting to attack and solve problematic situations. The MTDP model presented was developed from the experience obtained in about a hundred projects carried on in four years operations.
\end{abstract}

\section{Keywords}

- management of technology development projects model

- linking University-Enterprise

\section{El modelo empírico básico de la Gestión Tecnológica de Proyectos}

En la figura 1 se presentan las etapas del modelo de Gestión Tecnológica de Proyectos (GT de P), que ha sido aplicado con éxito en el Centro de Ciencias Aplicadas y Desarrollo Tecnológico (CCADET) de la Universidad Nacional Autónoma de México, (UNAM) (Vega, 2006).

Cada fase del modelo está conformada por diversas actividades no secuenciales. En la práctica se ha demostrado que es posible desarrollar diversas actividades en paralelo, por lo que frecuentemente se genera un corrimiento en el tiempo. El asunto tiende a complicarse un poco más cuando se desarrollan varios proyectos a la vez, ya que se requiere el desarrollo de múltiples actividades en forma simultánea. Cuando la GT de P se realiza para proyectos empresariales destinados al desarrollo de nuevos productos, el proceso de GT de P queda inmerso dentro del proceso de innovación continuo (Hughes et al., 1992). Los procesos incluidos en ca-

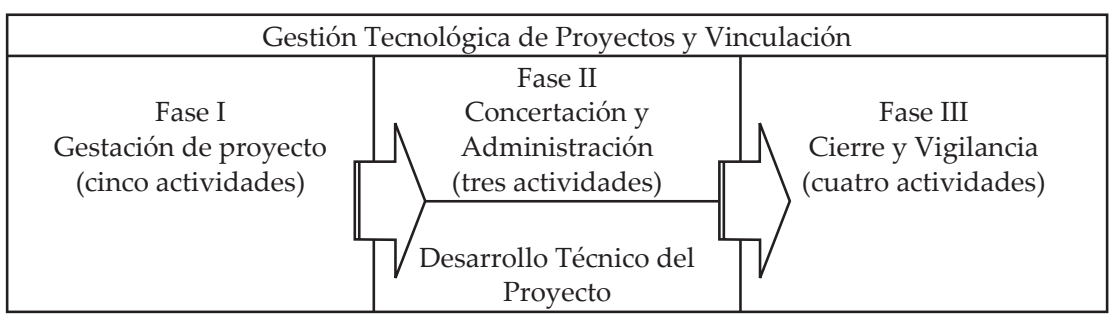

Figura 1. Etapas en la GT de Proyectos (GT de P) da una de las fases del modelo de GT de P, se describen a continuación.

\section{Fase I. Gestación del proyecto de DT}

El enfoque de "solución de problemas", (problem solving), seguido en el CCADET, coincide con el propuesto por Marquis, citado por Cusumano et al. (1992), en el que se requiere entender las necesidades del usuario para entonces tratar de obtener soluciones específicas por medio de las capacidades de desarrollo de tecnologías de los grupos de investigación aplicada y desarrollo tecnológico del Centro. En la gestación de todos los proyectos de desarrollo tecnológico hay una serie de etapas que se describen a continuación.

Identificación de la necesidad o la demanda (DEM)

El Desarrollo Tecnológico (DT), es un proceso creativo a través del cual se diseñan productos o procesos para satisfacer alguna necesidad. El resultado o producto del

DT son los objetos, dispositivos o paquetes tecnológicos. Dentro de su ciclo de vida, los productos tecnológicos se conceptualizan, se definen sus especificaciones, se implementan y se mantienen (Sage, 1992).

Los productos tecnológicos tienen características físicas y funcionales específicas (Kroes, 1998). El patrocinador es quien generalmente demanda un proyecto de DT y 
tiene una primera idea de las características que desea como producto. Sin embargo, no siempre es sencillo identificar cuál es la necesidad o demanda a cubrir, sino quién la tiene. Una de las funciones principales de quien realiza la Vinculación y Gestión Tecnológica es realizar esta identificación, lo cual no es un proceso sencillo.

La diversidad de demandas y solicitudes hace que el inicio de la fase I de gestación se presente con un "frente difuso" (Fuzzy Front End). Al iniciar el proceso de detección de la demanda, se requiere un tamizado de proyectos tal y como se muestra en la figura 2. En la práctica resulta que de cada diez proyectos que aparentemente se requieren en el mercado, cuya demanda se ha identificado por medio de diversos procedimientos y medios de comunicación, como son llamadas telefónicas, correos electrónicos, contactos personales, etcétera, sólo entre 30\% y 40\% son proyectos de DT que cuentan con un patrocinador potencial con desarrollo aparentemente factible en el CCADET. Cabe aclarar que en el Centro se desarrollan productos tecnológicos como instrumentos de medición, en los cuales se integran tecnologías de electrónica, mecánica, mecatrónica, óptica, acústica, microondas y software. El efecto de "embudo" no es privativo de este tipo de tecnologías, ya que por ejemplo, en el caso de la industria Química Farmacéutica, se requiere probar más de 5000 compuestos para producir una sola droga comercializable, lo que se realiza a través de entre 40 a 50 proyectos diferentes (Jacob et al., 2003).

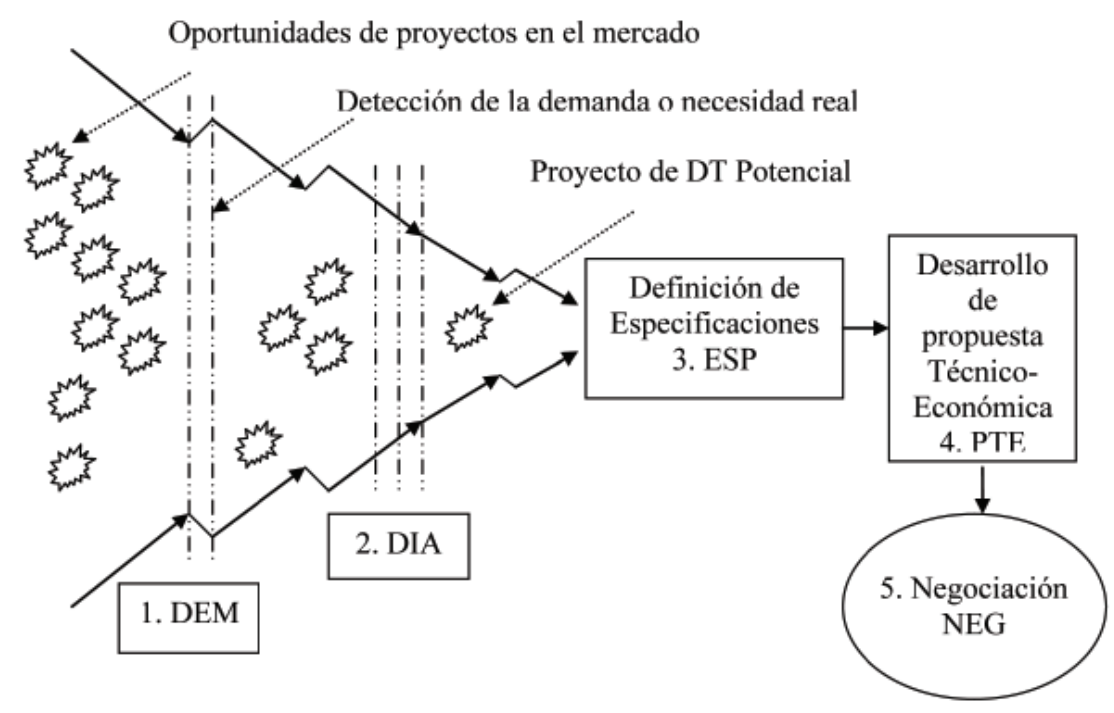

Figura 2. Tamizado del frente difuso y efecto de "embudo" para Proyectos en la Fase de Gestación. (Modificado de Zien et al., 1997)

\section{Diagnóstico capacidades internas (DIA)}

El siguiente proceso de la fase de gestación es el diagnóstico de capacidades internas; es decir, la formación del equipo de trabajo que realizará el proyecto. Los equipos de trabajo que realizan actividades de desarrollo tecnológico tienen que ser multifuncionales para ser efectivos (Sundstrom et al., 1990). En la Figura 2 este proceso se identifica como una barrera, debido a que no todos los proyectos detectados en DEM tienen viabilidad para ser realizados por los distintos laboratorios del CCADET. La realización de este proceso requiere conocer, en lo general, las capacidades de los académicos y las líneas de investigación y DT que desarrollan los distintos laboratorios y departamentos del centro. En el proceso DIA debe de realizarse una identificación y concertación plena del equipo de trabajo, ya que aunque un proyecto sea en apariencia realizable, no necesariamente podrá insertarse dentro de los planes de trabajo anuales de los laboratorios.

Por esta razón no es sino hasta después del proceso de diagnóstico DIA que se detectan los proyectos de DT potenciales factibles. El porcentaje de los mismos resulta ser de máximo un $15 \%$ de los proyectos detectados en el frente difuso como máximo.

\section{Definición de especificaciones (ESP)}

En general, todo proyecto de diseño usualmente tiene algún nivel de ambigüedad y es impredecible (Denton, 1997). Desde la perspectiva del "Aprendizaje Organizacional", un requisito indispensable es evitar la ambigüedad (Adams et al., 1998). Por eso, para que la organización aprenda la mejor forma de realizar proyectos de desarrollo tecnológico bajo demanda, es necesario definir las especificaciones lo mejor posible y esta actividad se convierte en uno de los procesos clave de la fase de gestación de proyectos. Este proceso requiere una comunicación intensa entre el patrocinador y el o los posibles académicos de los laboratorios interesados en el desarrollo y el área de vinculación y gestión tecnológica. De hecho, es aquí donde se inicia el proceso de DT, ya que el proceso ESP es en cierta manera, la definición concreta del problema. En este momento, se definen las ca- 
racterísticas deseadas del artefacto, proceso o producto tecnológico; también se definen los requerimientos comerciales y las especificaciones técnicas.

Planteamiento de la propuesta

técnico-económica (PTE)

Con la especificación técnica básica del proyecto y la definición del equipo de trabajo que lo desarrollará, se pueden estimar los materiales, las herramientas, los equipos necesarios y el tiempo esperado para su conclusión. Con los elementos anteriores existen las condiciones para desarrollar una propuesta técnico-económica, la cual deberá incluir los costos de los recursos humanos, los materiales consumibles, el equipo, viáticos, los gastos de transporte y cualquier otro gastos que sea necesario para llevar cabo el proyecto de DT. Por otra parte, se deben agregar los indirectos institucionales para llegar al precio del proyecto que será presentado al patrocinador.

\section{Negociación (NEG)}

La propuesta técnico-económica se presenta al patrocinador y con esto, se inicia un proceso evidente y fundamental de negociación para conciliar los términos comerciales, el tiempo de desarrollo y las especificaciones técnicas del proyecto con las aportaciones financieras que el patrocinador está de acuerdo en otorgar.

En diferentes investigaciones relativas a los factores de éxito de los proyectos de investigación y desarrollo ( $R \& D$, por sus siglas en inglés) en países desarrollados, no se considera la negociación como uno de los factores principales en el éxito de los proyectos de desarrollo de nuevos productos o tecnologías. Por ejemplo, en un estudio realizado por Astebro (2004) en más de 561 proyectos empresariales de baja inversión relativa, para el desarrollo de nuevos productos, se encontró que generalmente las variables centrales son una mezcla entre la utilidad esperada en términos de la oportunidad tecnológica y el riesgo de desarrollo, bajo ciertas condiciones de apropiabilidad patrimonial. Dependiendo del tipo de industria cobran importancia otro tipo de variables; por ejemplo, en la industria automotriz son importantes aspectos tales como el desempeño de las partes o la disponibilidad de proveedores (Cusumano, 1992).

Haciendo una revisión de más de sesenta artículos en la literatura en campos relacionados, (Balachandra et al., 1997), encontraron que las variables fundamentales que agrupan los factores de éxito son: el mercado, el medio ambiente, la tecnología y la organización en la que se desarrolla el proyecto. En los más de 120 factores de éxito identificados en sus estudios, nunca se menciona a la negociación como factor determinante, pero sí se menciona la efectividad del gerente de proyecto, la habilidad en la administración, el contar con un gerente de proyectos calificado y la comunicación. Sin embargo, es muy claro que en los países en vías de desarrollo, la etapa de negociación es uno de los principales factores de éxito, ya que si no se logra un acuerdo en ésta, simplemente el proyecto de DT queda suspendido. Posteriormente, veremos que a lo largo de las Fases II y III de la GT de P existen otros momentos cruciales de negociación.

En la figura 3 se muestran las diferentes etapas de la fase de gestación de la GT de P. Esta fase parte de la llegada del patrocinador que solicita el desarrollo del proyecto y culmina en el acuerdo al que se llega entre las partes, respecto a los términos y condiciones de la propuesta técnico-económica.

\section{Fase II. Concertación y administración del proyecto de DT}

Cuando se ha logrado un buen acuerdo después de la negociación del proceso (1.5 NEG), es el momento de llegar a la firma de instrumentos contractuales que garanticen para ambas partes la consecución del desarrollo en tiempo y forma, así como el pago oportuno del financiamiento. En los instrumentos contractuales de concertación y administración queda plasmada la planificación jurídica y administrativa del proyecto de DT. También se define quiénes serán los participantes del equipo de desarrollo y el calendario para hacer el seguimiento de todas las actividades concertadas para llegar a los entregables que se definan.

\section{Desarrollo de instrumentos contractuales de acuerdo (CON)}

Una vez que se llega al acuerdo en los términos técnicos y económicos del proyecto de DT con el patrocinador, llega el momento de decidir cuáles y de qué tipo serán los instrumentos contractuales de acuerdo. Aunque generalmente la UNAM trabaja por medio de convenios de colaboración, existen otros instrumentos de concertación como las cartas compromiso, las cartas de intención, los convenios de confidencialidad, las órdenes de servicio, los convenios de desarrollo tecnológico, los de licenciamiento y transferencia de tecnología, etcétera. Esta es la parte del engranaje legal. En la definición de los términos y clausulado de los instrumentos, participan la Coordinación de Vinculación del CCADET y la Secretaría Jurídica de la Coordinación de la Investiga- 


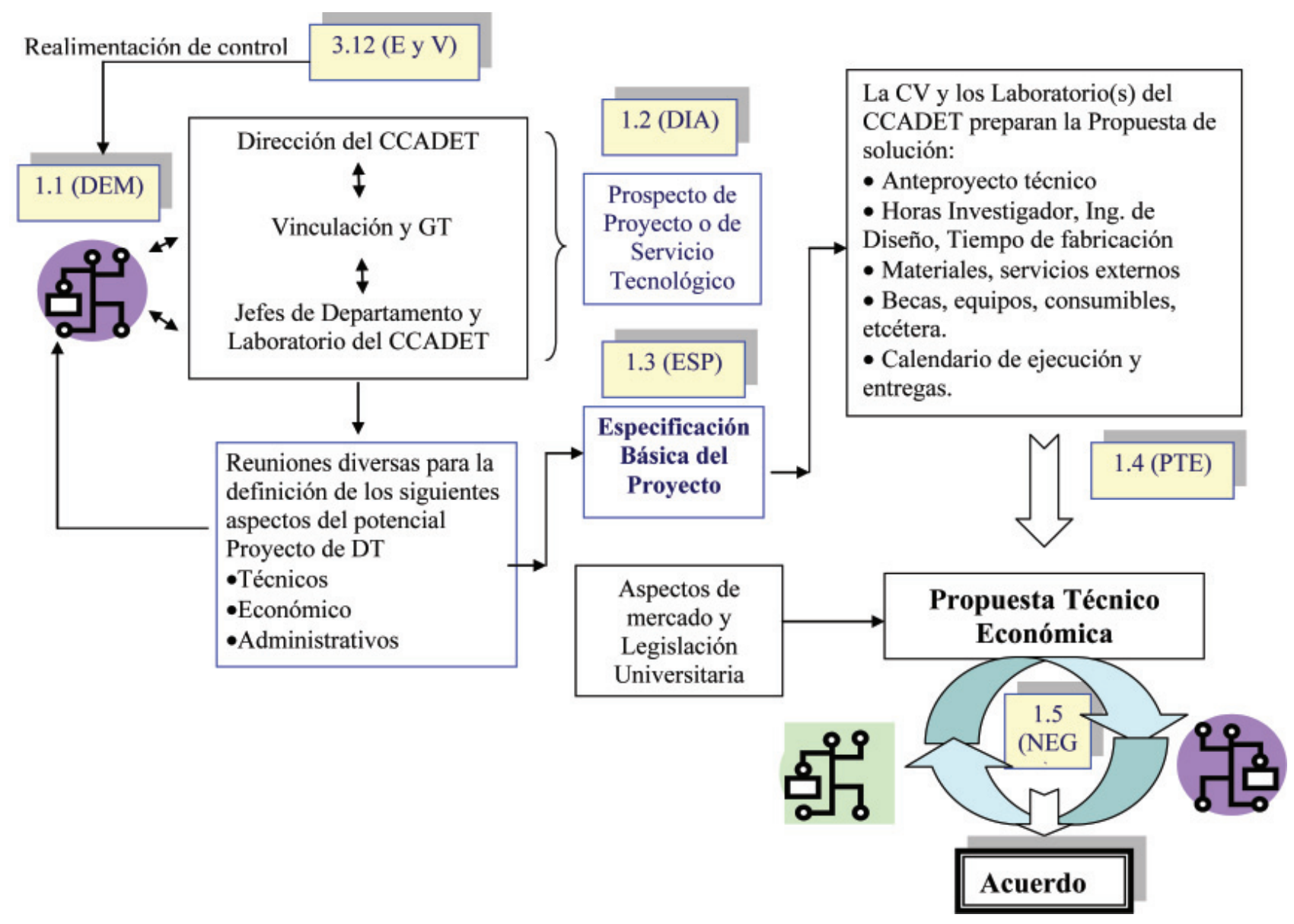

Figura 3. Diagrama de flujo de la fase I del modelo de GT de P

ción Científica, quien trabaja bajo los lineamientos jurídicos del Abogado General de la UNAM y los departamentos jurídicos y legales de la contraparte. Cuando se llega a un acuerdo, con los documentos legales firmados, se obtiene el pago de los anticipos, con lo que se da inicio a la ejecución técnica del proyecto de DT. Cabe observar que desde el punto de vista de la GT de P para llegar a este punto se requiere el antecedente de seis procesos diferentes y dado que en muchos casos nunca se llega a la firma de acuerdos, frecuentemente se hace una inversión de tiempo y esfuerzo infructuosa.

\section{Planeación y seguimiento administrativo (P y SA)}

Con los instrumentos contractuales firmados y el pago de anticipos se realiza la apertura del proyecto, el cual es un documento interno que una vez aprobado guiará la logística interna de ejecución. Este documento de apertura debe contar con la firma de enterado del coordinador de vinculación y la aprobación del director. La apertura del proyecto es el documento de control clave que representa la planificación de recursos de la institución (IMNC, 2003), por lo que debe ser firmado por el responsable técnico del proyecto, por el jefe de departamento respectivo y por el director del centro. La Secretaría Administrativa del Centro lo debe procesar y dar entrada al sistema administrativo generando las partidas de presupuesto para su ejercicio inmediato. La evaluación de proveedores, las contrataciones y los procesos de compra se deben realizar por el personal de la Secretaría Administrativa del CCADET, quienes deberán hacer la planeación, evaluación, documentar y llevar el control de los contratos (IMNC, 2003).

\section{Administración tecnológica del proyecto (AT)}

Este es un proceso fundamentalmente de control y comunicación, requiere el registro cuidadoso de todos los acuerdos que se toman en las minutas de las juntas de trabajo y de la verificación de su cumplimiento en 
tiempo y forma. En este proceso también se generan los "Libros del proyecto" y se documentan todas las notas informativas o transmisiones de documentos. Se debe llevar un control cuidadoso de la ruta crítica, así como de las fechas de entrega de las distintas fases del proyecto técnico y del cumplimiento de los entregables acordados en los convenios de concertación.

Esta fase de gestión es otro de los procesos relacionados con el tiempo, con el costo y con la comunicación (IMNC, 2003).

El administrador del proyecto también debe generar e incluir documentación sobre la estrategia de desarrollo y la estrategia de propiedad intelectual que se llevará a cabo más adelante. En la figura 4 se muestra el diagrama de bloques de las diferentes actividades que constituyen la fase II de la GT de P.

\section{Fase III. Cierre y vigilancia}

Cuando termina la ejecución técnica del proyecto de DT, desde el punto de vista de la gestión tecnológica, todavía falta una fase final que inicia con los entregables que se obtienen del proceso de administración tecnológica. Es necesario vigilar el adecuado empaque- tamiento de la tecnología, hacer la entrega formal de los paquetes tecnológicos y obtener las cartas de aceptación y finiquito por parte de los clientes.

\section{Cierre del proyecto $(\mathrm{CP})$}

Es bien sabido para los tecnólogos industriales que es más fácil iniciar un proyecto, que terminarlo (Lieb, 1998). Para cerrar un proyecto, hay que terminarlo bien. Por lo tanto, esta actividad tiene como foco fundamental la vigilancia de entregables, la cual es muy importante, ya que consiste en la entrega de los prototipos desarrollados, los manuales de operación, de servicio y, en su caso, de fabricación. Frecuentemente, se incluyen cursos de capacitación para operadores y esquemas de garantía de refacciones y existencia de partes en el mercado nacional. Lograr la aceptación definitiva de un proyecto de DT no siempre es sencillo, hay que recordar que se trata de prototipos en alguna de sus fases de desarrollo, que partieron de una especificación que ostentaba un cierto grado de ambigüedad; por lo que en principio, todos los prototipos siempre pueden mejorarse. Además, también existe la posibilidad de que el tiempo de realización de las fases I y II del proyecto se
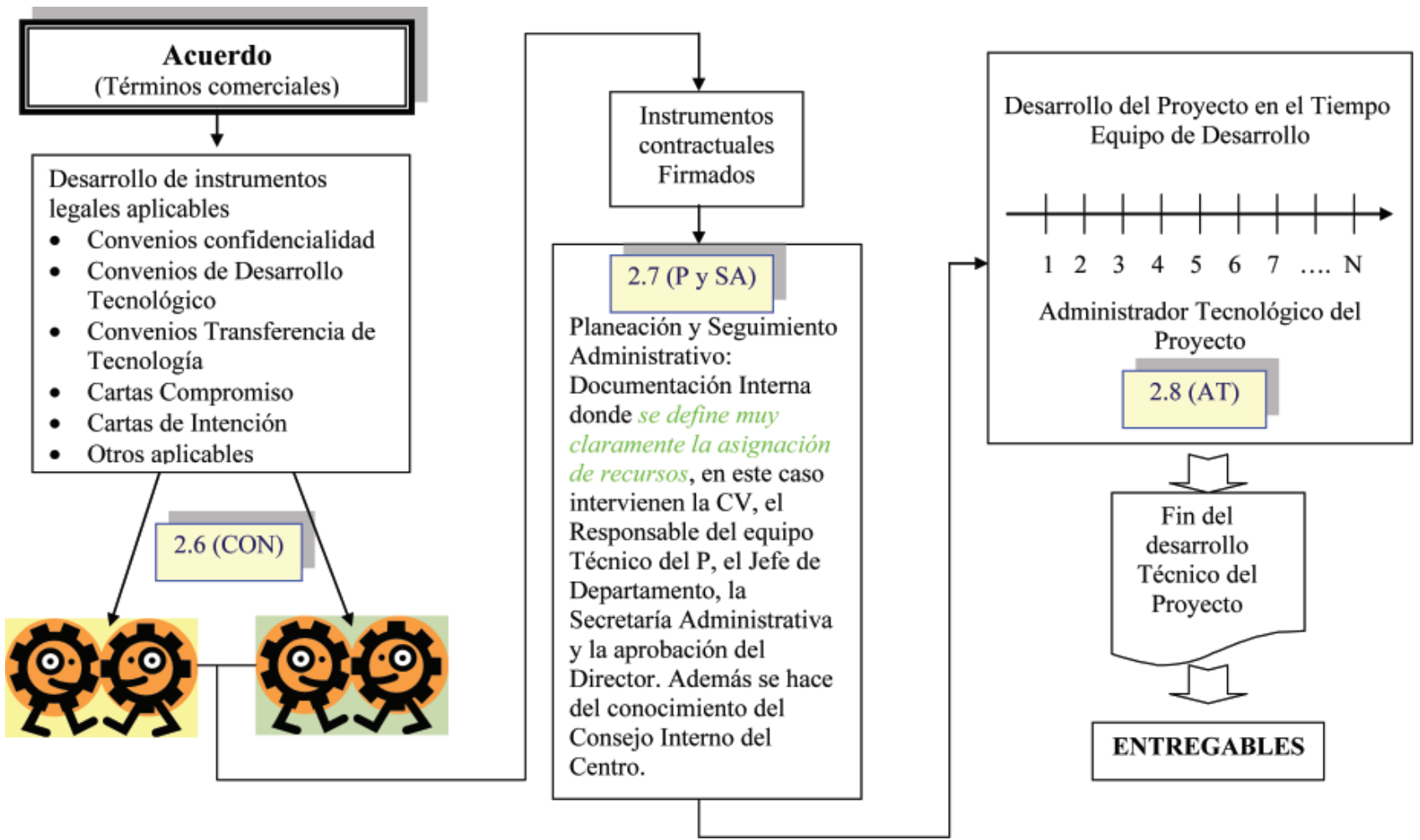

Figura 4. Diagrama de flujo de la fase II del modelo de GT de P 
haya alargado de tal forma, que el ciclo de vida del producto tecnológico resultado del proyecto esté llegando a su término.

El cierre de un proyecto es tan o más importante que todas las etapas anteriores de la GT de P. Su importancia está perfectamente definida en el punto de la norma aplicable (IMNC, 2003), ya que se debe integrar la documentación legal y del paquete tecnológico.

\section{Propiedad intelectual (PI)}

Otro proceso muy importante de la GT de P es el relativo a la obtención de títulos de propiedad intelectual. Este es un aspecto de mucha relevancia, ya que por ley, corresponde a las universidades por su papel patronal la propiedad patrimonial de todos los desarrollos en los que participa personal que está empleado en cualquiera de sus dependencias o entidades.

Para el estudio, evaluación y obtención de certificados y títulos aplicables se requiere personal especializado, profundamente capacitado y con experiencia en patentes, modelos de utilidad, diseños industriales, derechos de autor y secretos industriales.

Tal vez la parte más importante de la gestión de la propiedad intelectual es la definición de las estrategias, las cuales deben estar alineadas con las políticas institucionales propias de la dependencia y deben pasar por un riguroso examen de costo/beneficio.

\section{Licenciamientos (L)}

Una vez terminado un proyecto y obtenidos sus resultados tecnológicos, es necesario cerrarlo a través de la firma de un convenio de transferencia de tecnología (CTT) o de un convenio de licenciamiento (CL): esto dependerá de los términos de propiedad intelectual que se hayan acordado en el convenio de desarrollo. Si los resultados no son objeto de solicitud de títulos de propiedad intelectual o bien, si se acordó que la propiedad intelectual correspondería al patrocinador, para cerrar el proyecto es suficiente con la firma de un CTT. Si por estrategia se decide que deben solicitarse títulos de patente o certificados de derechos de autor para los resultados tecnológicos, debe procederse con los trabajos de redacción y presentación de solicitudes ante las autoridades correspondientes.

Una vez obtenidos los títulos de propiedad intelectual, si la organización patrocinadora manifiesta su interés en el escalamiento y comercialización de la tecnología, tiene derecho de prioridad para licenciar la tecnología. En este caso el CIT que se firme puede incluir los términos de licenciamiento. Por otra parte, si el patrocinador manifiesta expresamente que no tiene interés en la explotación de la tecnología, entonces la universidad queda en total libertad de negociar el licenciamiento con alguna otra empresa u organización interesada.

Para definir los términos del CL se requiere la aplicación de técnicas de evaluación tecnológica mediante las cuales es posible definir el valor comercial de la tecnología y, en consecuencia, el porcentaje de regalías que se cobrarán, la periodicidad de las mismas, la vigencia del CL, los términos de exclusividad y el alcance de la propiedad patrimonial transferida.

\section{Evaluación y vigilancia (E y V)}

El proceso final de la GT de $\mathrm{P}$ tiene que ver con la determinación del impacto económico y/o social y con la gestión del conocimiento. Además de la revisión de los costos de desarrollo y de administración en los que incurrió la organización para la consecución del producto tecnológico, hay que cuantificar la forma en que el desarrollo del proyecto contribuyó a la generación de competencias críticas. Como un primer paso, hay que identificar cuáles fueron los laboratorios involucrados y el personal académico que participó específicamente en el proyecto, así como desarrollar un estudio comparativo de los diferentes proyectos de DT que se llevaron a cabo durante un año específico, durante un lustro o una década. Esto permite identificar el tipo de proyectos más exitosos y de mayor impacto social y económico para favorecer el desarrollo académico de los grupos en términos de lograr las expectativas de la visión de largo plazo del Centro. Posteriormente, hay que vigilar la forma en que las empresas licenciadas llevan a cabo la promoción y difusión de las tecnologías y, si eventualmente las mismas se convertirán en innovaciones. El número de proyectos que constituyan la solución a problemas específicos detectados en el proceso de detección de demanda permitirá identificar y cuantificar la forma en que el CCADET ha cumplido con su misión de coadyuvar en la solución de problemas nacionales y hará positiva la balanza de su contabilidad social.

En la figura 5 se muestra el diagrama de bloques de las diferentes actividades que constituyen la fase III de la GT de $P$.

\section{Conclusiones}

Frecuentemente la administración de proyectos de I\&DT se ha asociado con las actividades de escrutinio, evaluación, selección, elaboración de presupuestos y calendarios, así como el control de los proyectos. Liberatore \& Titus, citados por Vislosky (2000). Esto puede 


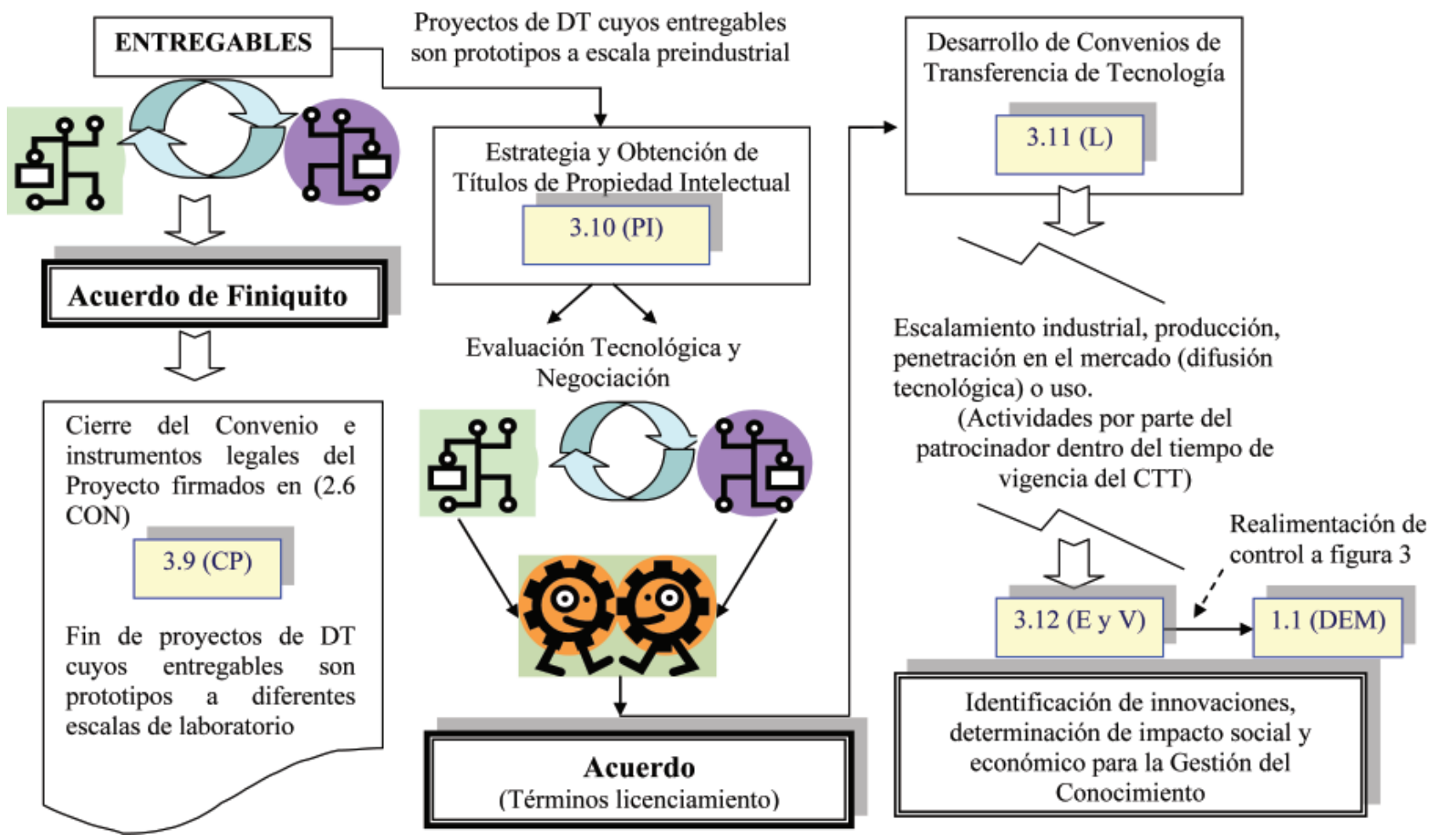

Figura 5. Diagrama de flujo de la fase III del modelo de GT de P

aplicarse para organizaciones que realizan I\&DT y operan en economías del primer mundo, ya que cuentan con una mayor división de áreas operativas y por lo tanto, las funciones se distribuyen. En los centros de I\&DT que se encuentran operando en economías del tercer mundo, particularmente en el caso mexicano, las funciones de quienes ejecutan la GT de P, se incrementan.

En éste último caso, el intenso flujo de información queda evidente en el modelo integrado de malla cerrada de la GT de P presentado en la figura 6, en la que se incluye el lazo de realimentación que va del proceso final al proceso inicial y que sirve para regular y conducir el comportamiento de la organización a través de la gestión del conocimiento.

El modelo conceptual aquí presentado, se sustenta en la validación empírica de la gestión de cerca de un centenar de proyectos de desarrollo tecnológico realizados durante los años 2002 a 2006 en el Centro de Ciencias Aplicadas y Desarrollo Tecnológico de la UNAM. Todos los proyectos tuvieron duraciones diversas, con una vigencia contractual que osciló desde tres meses, hasta tres años y fueron desarrollados por equipos multidisciplinarios de académicos adscritos a diversos laboratorios del Centro. Cabe aclarar que en algunos casos aunque la vigencia de los convenios fue de tres años, el tiempo total desde el proceso de demanda hasta el proceso de licenciamiento tomó cerca de diez años. Por otra parte, tenemos la referencia de que algunos proyectos de DT con resultados innovadores han tomado más de quince años desde el proceso hasta el proceso de evaluación y vigilancia.

En muchos de los proyectos gestionados se realizaron actividades simultáneas; es decir, mientras en algunos casos se podía estar iniciando la gestión de un proyecto, en otros se desarrollaba la fase de firma de convenios y otros más se encontraban en periodo de cierre o licenciamiento.

En términos generales, los proyectos de DT terminan en el proceso de cierre del proyecto de la fase III. Los paquetes tecnológicos entregables pueden encontrarse en alguna de las diferentes escalas de integración de laboratorio. No hay regla, la escala de integración depende del tipo de tecnología y de los alcances definidos para el proyecto. En esos casos, frecuentemente se realizan proyectos recursivos para mejorar la versión de la escala del DT. Por esta razón, en el modelo final presentado en la figura 6, se muestra una realimentación del proceso hacia el proceso inicial. Cuando el DT ya ha alcanzado una escala pre-industrial generalmente hay resultados de propiedad industrial y se requiere la cele- 


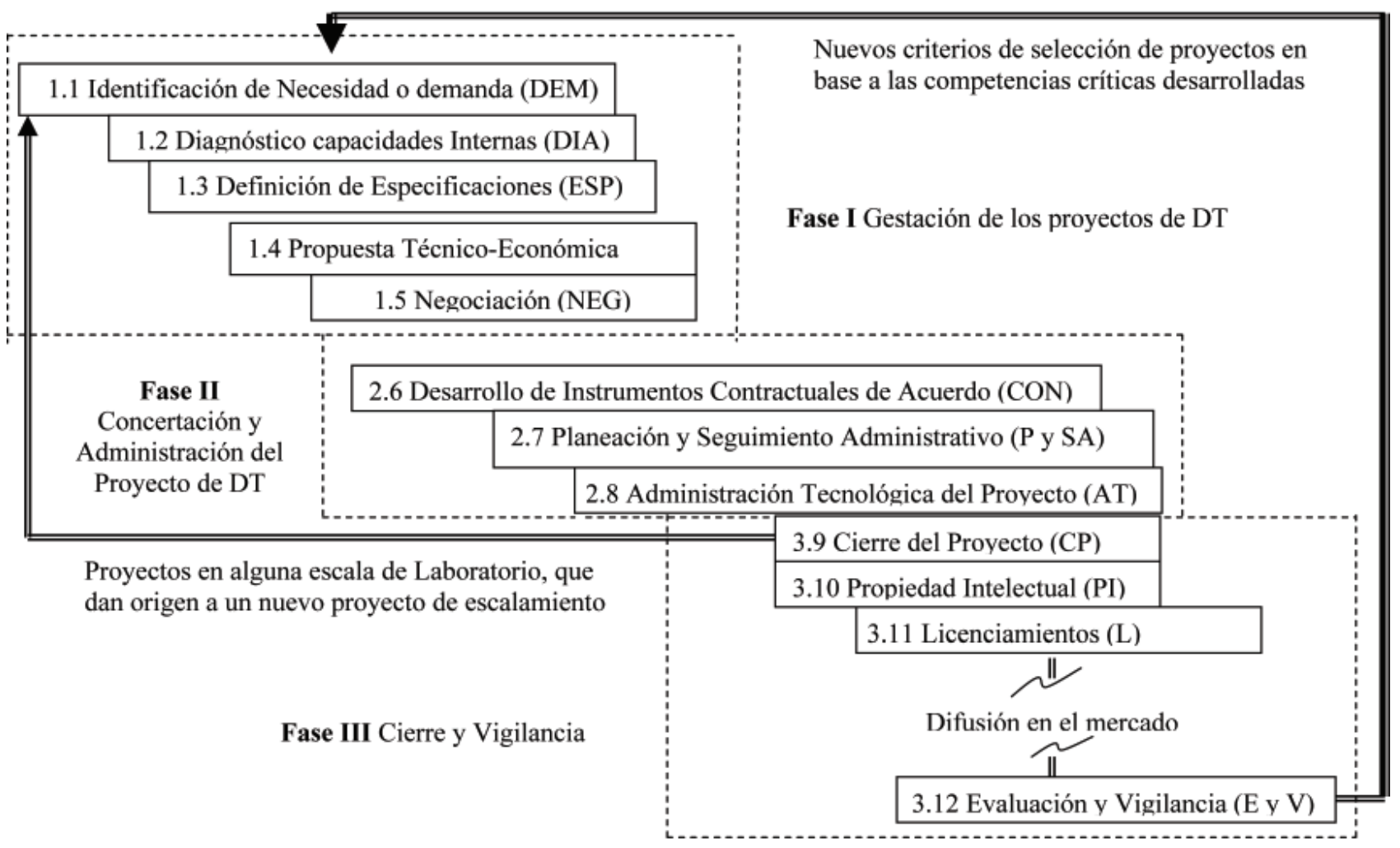

Figura 6. Modelo simplificado de malla cerrada de la GT de P

bración de convenios de transferencia tecnológica en los que se licencian estos derechos a la empresa patrocinadora. Una vez liberada la tecnología corresponde a la empresa hacer las gestiones para producir el dispositivo e introducirlo al mercado, generando un impacto o aportación económica y/o social que así se convierte en una innovación. A través del proceso de evaluación y vigilancia, es posible determinar si el CCADET cumplió con sus objetivos y con su misión organizacional.

Según Lederman (2004), economista en jefe para América Latina y el Caribe del Banco Mundial, los Centros de I\&DT deben encaminar sus esfuerzos hacia la innovación que se transforme en productos potencialmente comercializables. Los proyectos de desarrollo tecnológico son la génesis de los proyectos de innovación (Marinova, 2003). Indudablemente la aplicación de las técnicas de gestión tecnológica de proyectos (GT de P), es a su vez, la génesis de la innovación organizacional, la cual debe ser concebida como un proceso de aprendizaje organizacional en el que la información se debe adquirir, diseminar y utilizar en forma rutinaria (Adams, 1998).

Como todos los modelos empíricos, el nuestro puede seguramente mejorarse a partir de la información que aporten nuevos casos de gestión. Como trabajo futuro, a partir de este modelo se desarrollará una metodología para determinar el costo intangible total de la GT de P en los proyectos de desarrollo tecnológico. Para finalmente valorar y cuantificar la aportación de valor intangible de la negociación, en la GT de P.

\section{Referencias}

Adams M.E, Day G., Dougherty D. Enhancing New Product Development Performance: An Organizational Learning Perspective. Journal of Product Innovation Management, 15:403-422. Elsevier Science Inc. 1998.

Astebro T. Key Success Factors for Technological Entrepreneur's R\&D Projects. IEEE Transactions on Engineering Management, 51(3):314-321, August, 2004.

Balachandra R., Friar J. Factors for Success in R\&D Projects and New Product Innovation: A contextual Framework. IEEE Transactions on Engineering Management, 44(3):276-287, August, 1997.

Cusumano M., Nobeoka K. Strategy, Structure and Performance in Product Development: Observations from the Auto Industry. Research Policy, 2:265-293, Elsevier North Holland. 1992. 
Denton, H.G. Multidisciplinary Team Based Project Work: Planning Factors. Design Studies, 18:155-170. Elsvier Science Ltd. Great Britain. 1997.

Hughes D., Chafin D. Turning New Product Development into a Continuous Learning Process. Journal of Product Innovation Management, 13:89-104. Elsevier Science Inc. 1996.

Instituto Mexicano de Normalización y Certificación, IMNC. Norma PROY-NMX-CC-10006-IMNC-2003. Sistemas de gestión de la calidad: Directrices para la gestión de la calidad de los proyectos. México. 2003.

Jacob W.F., Kwak Y.H. In Search of Innovative Techniques to Evaluate Pharmaceutical R\&D Projects. Technovation, 23:291-296. Elsevier Science Ltd. 2003.

Kroes P. Technological Explanations: The Relation Between Structure and Function of Technological Objects. Philosophy and Technology. Society for Philosophy and Technology. 3(3). Spring, 1998.

Lederman D. Innovación en México: Síntesis. documento mimeografiado. Oficina del Economista en Jefe para ALC, Banco Mundial, Washington, DC. 2004.

Lieb E.B. How Many R\&D Projects to Develop? Transactions on Engineering Management, 45(1):73-77. IEEE. 1998.
Marinova D., Phillmore J. Models of Innovation. The International Handbook of Innovation. Edited by Larisa V. Shavinina. Elsevier Science Ltd. 2003. Pp. 44-53.

Sage A.P. Systems Engineering. John Wiley \& Sons, Inc. New York. 1992. ISBN: 0-47-53639-3. 606 p.

Sundstrom E., De Meuse K., Futrell D. Work Teams, Applications and Effectiveness. American Psychologist, 45(2):120-133. The American Psychologist Association, Inc. USA, 1990

Vega G.L.R. Modelo del ciclo de vida de un proyecto de gestión tecnológica y vinculación en un centro de I\&D universitario. Memorias del $1^{\text {a }}$ Congreso Iberoamericano de Ciencia, Tecnología, Innovación y Sociedad (CTI-S). Palacio de Minería, México, Junio, 2006.

Vislosky D.M., Fischbeck P.S. A Mental Model Approach Applied to R\&D Decision Making. International Journal of Technology Management, 19(3-5):453-471. Inderscience Enterprises Ltd., 2000.

Zien K.A., Buckler S.A. From Experience Dreams to Market: Crafting a Culture of Innovation. Journal of Product Innovation Management, 14(3):284-287, August, 1997.

\section{Semblanza del autor}

Luís Roberto Vega-González. Es ingeniero mecánico electricista por la Facultad de Ingeniería de la Universidad Nacional Autónoma de México (UNAM). Obtuvo la maestría en ingeniería de sistemas en el área de planeación por la División de Estudios de Posgrado de la Facultad de Ingeniería, UNAM y la maestría en administración de las organizaciones por la División de Estudios de Posgrado de la Facultad de Contaduría y Administración de la UNAM. Se especializó en gestión tecnológica por el Centro de Innovación Tecnológica y la Facultad de Contaduría y Administración de la UNAM. Durante más de veinte años colaboró en diversas firmas nacionales e internacionales desarrollando proyectos de ingeniería e integración tecnológica en las áreas de instrumentación, control y potencia. Desde 1993, es académico de tiempo completo de la UNAM donde ha colaborado en el Centro para la Innovación Tecnológica, el Instituto de Ingeniería y el Centro de Instrumentos. Actualmente es coordinador de vinculación y gestión tecnológica del Centro de Ciencias Aplicadas y Desarrollo Tecnológico (CCADET). Ha conducido negociaciones, convenios, contratos y realizado actividades de administración tecnológica para cerca de dos centenares de proyectos en la UNAM. Es autor de aproximadamente un centenar de diferentes productos académicos en sus diferentes modalidades en los temas de su especialidad 